\title{
The association between detected drug resistance mutations and CD4 cell decline in HIV-positive individuals maintained on a failing treatment regimen
}

[RUNNING HEAD]: HIV drug resistance and CD4 counts

SCHULTZE, Anna ${ }^{1}$; PAREDES, Roger ${ }^{2}$; SABIN, Caroline ${ }^{1}$; PHILLIPS, Andrew N ${ }^{1}$; PILLAY, Deenan ${ }^{3,4}$; MACKIE, Nicola ${ }^{5}$; CASTAGNA, Antonella ${ }^{6}$; CHADWICK$^{7}$, David; FALCONER, Karolin ${ }^{8}$; GERETTI, Anna Maria ${ }^{9}$; POST, Frank $A^{10}$; HILL, Teresa ${ }^{1}$; KIRK, Ole ${ }^{11}$; POZNIAK, Anton ${ }^{12}$; NELSON, Mark ${ }^{12}$; TOSTEVIN, Anna $^{1}$; DUNN, David ${ }^{1,13}$; LUNDGREN, Jens ${ }^{11}$ and COZZI-LEPRI, Alessandro ${ }^{1}$

1. Department of Infection and Population Health, UCL, UK

2. Institut de Recerca de la SIDA-IrsiCaixa, Badalona, Spain

3. Division of Infection and Immunity, UCL, London, United Kingdom

4. Africa Centre for Population Health, University of KwaZulu-Natal, South Africa

5. Imperial College, London, UK

6. Università Vita-Salute San Raffaele, Milano. Italy

7. James Cook University Hospital, Middlesbrough, UK

8. Department of Infectious Diseases, Karolinska University Hospital, Stockholm, Sweden

9. University of Liverpool, Liverpool, UK

10. Kings College Hospital, London, UK

11. Department of Infectious Diseases, CHIP, Section 2100, Rigshospitalet, University of Copenhagen, Denmark

12. Chelsea and Westminster Hospital, London, UK

13. MRC Clinical Trials Unit, UCL, London, UK

Correspondence to: Anna Schultze, HIV \& Biostatistics Group, Department of Infection and Population Health, UCL Royal Free Campus, NW3 2QG, London, UK

Email: a.schultze@ucl.ac.uk

Word Count: $3785 / 4000$

References: 44

Inserts (Tables and Figures): 5

Appendices: 5 docx files

Abstract: $224 / 250$ 


\section{Abstract}

2

Objectives: To analyse the effect of drug resistance mutations (DRM) on CD4 cell trends in HIVpositive people maintained on virologically failing antiretroviral therapy (ART).

Patient and Methods: Individuals from two large cohorts experiencing virological failure (VF) while maintained on ART with $\geq 1$ CD4 count and $\geq 1$ resistance test were included. CD4 cell slopes were estimated using linear mixed models. Principal component analysis (PCA) was used to assess the effect of clusters of mutations, defined using extracted component based scores from the PCA, on CD4 cell decline.

Results: 5,357 individuals contributing 7,661 VF episodes were included: any DRM were detected in $88.8 \%$ of episodes. After adjustment, CD4 counts declined less steeply during episodes where DRM were detected compared to episodes with no DRM (difference=28 cells $/ \mathrm{mm}^{3} /$ year, $95 \% \mathrm{Cl}=18-39$, $p<0.001)$. Among individuals with at least one DRM, we found evidence that any nucleoside/nucleotide reverse-transcriptase inhibitor (NRTI) resistance, the reverse transcriptase (RT) mutations M184V, D67N and T215Y as well as the protease mutations V82A and I54V were associated with reduced CD4 cell declines. The detection of any non-nucleoside reversetranscriptase inhibitor (NNRTI) resistance, the RT mutations V179D and L74V were associated with steeper CD4 cell declines. The presence of some mutation patterns similar to the clusters identified by the PCA also affected the CD4 cell decline.

Conclusion: Detection of resistance and of certain DRM during VF of ART has a small but significant favourable effect on CD4 cell decline. 
27 Key Words: Drug Resistance, HIV infection, Viral Fitness, Linear Mixed Models, Principal Component 28 Analysis 


\section{Introduction}

Despite the success of antiretroviral therapy (ART) in lowering mortality of people living with HIV, some individuals still experience virological failure (VF) on their ART regimen. Given the large number of potential anti-HIV drugs available, an effective second- or third-line salvage regimen consisting of new drugs from different drug classes can generally be prescribed [1]. However, for a minority of individuals it is sometimes difficult to construct a salvage regimen because of extensive and complex resistance patterns or, in resource-limited settings (RLS), because of limited drug availability, infrequent or absent viral load monitoring and a consequent lack of timely switching. In these situations, clinicians may be forced to keep individuals on their failing regimen for extended periods of time $[2,3]$.

Individuals who are kept on a failing ART regimen can still derive clinical benefit from the treatment and may experience stable or increasing CD4 counts despite ongoing viral replication [4-6]. This has been hypothesised to reflect lower replicative capacity of viruses with drug resistance mutations (DRM), particularly for the mutation M184V selected for by lamivudine [7]. However, other factors such as reduced CD4 cell turnover and/or activation [8], the inhibition of CD4 cell apoptosis by protease inhibitors (PI) [9], ineffective replication in the thymus by PI resistant viruses [10] or residual antiviral activity [11] have also been proposed as potential explanations. Although increased CD4 counts despite ongoing viral replication have been observed in several studies $[5,12-$ 17], only a few large studies have investigated the effect of DRM on CD4 counts [18-20]. We used data from two large European cohorts of HIV-positive people receiving ART to evaluate the association between the detection of DRM and CD4 count changes in the presence of ongoing viral replication. 
Methods

\section{Study participants}

We included individuals from the EuroSIDA cohort [21] and the UK Collaborative HIV Cohort (UK CHIC) Study [22] (with linked resistance data from the UK HIV Drug Resistance Database (UK HDRD) [23] ) who had $\geq 1$ episode of VF ( 2 or more consecutive viral load (VL) measurements of $>500$ copies/mL while remaining on the same ART regimen) who had $\geq 1$ resistance test and $\geq 1$ CD4 count measured during these episodes of VF . Episodes of VF were defined as starting on the date of the first VL measurement $>500$ copies $/ \mathrm{mL}$, and as ending on the date of the first $V L$ measurement $\leq 500$ copies/mL or on the date when the ART regimen was changed (defined as either stopping or adding one or more drugs). Participants could contribute multiple episodes of VF, and baseline was defined as the date of the first CD4 measurement of each episode.

\section{Exposure and outcome definitions}

We modelled CD4 counts linearly on a raw (i.e. cells $/ \mathrm{mm}^{3}$ ) scale. Resistance was classified using the IAS (2015), Stanford (2014), ANRS (2015) and Rega (2013) classification systems. We used the IAS list to classify minor PI mutations. Any resistance was defined as the detection of at least 1 DRM in reverse transcriptase (RT) or protease (PR) in either any of the 4 classification systems. Individuals with only minor PR mutations were considered as having no detectable resistance, and we only studied minor PR mutations when these were present with other DRM. The resistance categories that we studied were: nucleoside reverse transcriptase (NRTI) resistance, non-nucleoside reverse transcriptase (NNRTI) resistance, any protease inhibitor (PI) resistance (considering only major mutations) and any PI resistance (considering both major and minor mutations). We also evaluated the effect of individual mutations that were detected in $>1 \%$ of episodes. Resistance was presumed to be present from the start of the failure episode until the end, irrespective of the point during the episode at which it was detected by the genotypic test. 
As mutations can co-occur in patterns, we conducted a principal component analysis (PCA) to identify clusters of mutations in the RT and PR [24]. The details of the PCA are provided in the supplementary materials (S1). For simplicity, and to avoid capturing associations driven by temporal changes to the virus, we did not differentiate between different amino-acid substitutions in the same position for this part of the analysis. To study the effect of DRM clusters on CD4 count changes, we used extracted component-based scores from the PCA. These scores indicate how closely an individual's mutation pattern resembles that described by a given cluster. A high score indicates that the individual's mutation pattern is close to that of the cluster. After extracting the scores, we looked at whether individuals with high scores (defined as a score in the highest quartile) for a certain cluster had different CD4 count changes compared to those with lower scores.

\section{Statistical methods}

We compared CD4 cell slopes estimated using linear mixed models with random intercepts and slopes among individuals with any drug resistance to those without any detected resistance $[25,26]$. Among those with any resistance, we then evaluated the effect of class of resistance and individual DRM. The effect of PCA-derived scores on CD4 cell slopes was also evaluated among individuals with any resistance. Potential confounders were identified using previous publications and clinical knowledge, and the final model was adjusted for age (categorised into quartiles), hepatitis C status (yes/no/missing), risk group (Men who have sex with men/ Injecting Drug Use/ heterosexual/ other) and subtype (B/Non-B). A summary literature review undertaken before this study is provided in the supplementary materials (S2). We did not adjust for baseline CD4 counts, as this risks introducing bias $[27,28]$. VL can be considered both as a potential confounder and as a causal pathway variable, as the effect of any mutation on the CD4 cell decline may at least in part be mediated through changes in the VL. For this reason, we conducted step-wise adjustments as follows: 1) Exposure alone, 2) Model $1+$ age, hepatitis C status, risk group and subtype and 3) Model $2+$ VL. We adjusted 
105 for VL as a continuous, time-updated covariate on the $\log _{10}$ scale. For the baseline table we also

106 present data on the level of viral suppression. This was estimated by comparing current VL levels

107 with an individual's viral set point, which was defined as the mean of all pre-ART VL measures.

108

109 We adjusted the p-values derived from the analysis of individual DRM for multiple testing, as these

110 were chosen based on a prevalence threshold and not an a-priori hypothesis. To do this we used the

111 Benjamini-Hochberg procedure for correcting the false discovery rate (FDR) [29], which produces a

112 q-value representing the probability of a significant finding being a false positive. We used a q-value

113 threshold of 0.05 to indicate significant results. 


\section{Results}

\section{Characteristics of the study population}

117 A total of 5,357 individuals contributing 7,661 VF episodes were included in the analyses; 2,757 (36\%) from EuroSIDA and 4,904 (64\%) from UK CHIC. Individuals experienced a median of 1 (range=1-9) VF episodes, which lasted for a median of 5 (inter-quartile range [IQR]=2-13) months and contained a median of $3(\mathrm{IQR}=2-5) \mathrm{CD} 4$ measurements. The characteristics of the population at the start of each episode can be seen in the supplementary materials, S3. Although $15.6 \%$ of episodes occurred while individuals were on mono/dual therapy, the majority occurred while individuals were in receipt of more than 3 drugs. The most common type of cART was NNRTI based

124 (19.8\%). 56\% of episodes occurred while individuals were receiving lamivudine. The second most common drug was tenofovir (30\%) followed by indinavir (33\%). 1,338 different combinations of drugs were used, the most common being zidovudine, lamivudine and nevirapine. A description of all drugs utilised can be found in the supplementary materials (S4).

Any resistance was detected in 6,804/7,661 episodes (88.8\%). The prevalence of any NRTI resistance was $68.0 \%$, any NNRTI resistance $58.2 \%$ and any PI resistance (excluding minor PI mutations) $51.0 \%$. The prevalence of the 55 distinct RT mutations and $64 \mathrm{PI}$ mutations that were detected at a frequency of more than $1 \%$ can be seen in Figure 1. There were some differences in host and viral characteristics between episodes where resistance was detected compared to episodes where it was not (Supplementary materials, S3). Notably, episodes with no resistance had higher baseline viral load values $(p<0.001)$. The baseline viral load values were also more likely to be higher than setpoint estimates in episodes with no detected resistance $(p<0.001)$. However, there was only very weak evidence suggesting a difference in CD4 counts measured at the start of episodes ( $p=0.05)$. 
In univariable analysis, the estimated CD4 cell decline during episodes of VF was 20 cells $/ \mathrm{mm}^{3} / y e a r$

$140(95 \% \mathrm{Cl}=-22 ;-17)$ in the entire population. $\mathrm{CD} 4$ cell declines were less steep (although still notable) when time-updated VL levels were below pre-ART set-point values ( $p=0.008$; data not shown).

Figure 2 shows the estimated difference in annual CD4 cell decline according to the detection of any and class of resistance. CD4 counts declined less rapidly during episodes where drug resistance was detected compared to episodes where resistance was not detected, with an estimated difference of $28(18-39)$ cells $/ \mathrm{mm}^{3} /$ year $(-17[-20 ;-15] \mathrm{v}-46[-56 ;-36])$ cells $/ \mathrm{mm}^{3} /$ year, interaction $\left.\mathrm{p}<0.001\right)$.

These findings persisted after adjustment for the pre-specified confounders (difference=28 [17-39] cells $/ \mathrm{mm}^{3} /$ year, $p<0.001$ ) and after further adjustments for VL (difference=32 [21-42] cells $/ \mathrm{mm}^{3} /$ year, $\mathrm{p}<0.001$, Figure 2 ).

Among individuals with detected drug resistance, CD4 counts declined less rapidly during episodes where NRTI resistance was detected compared to episodes where NRTI resistance was not detected $\left(-15(-18 ;-12) v-40(-48 ;-33)\right.$ cells $/ \mathrm{mm}^{3} /$ year, interaction $\left.\mathrm{p}<0.001\right)$. Adjustment for pre-specified confounders and VL did not change the difference in CD4 cell slopes between those with and without NRTI resistance markedly (difference $=27$ [18-35] and 28 [20-37] cells $/ \mathrm{mm}^{3} /$ year respectively, Figure 2). There was also evidence that CD4 counts declined faster during episodes where NNRTI resistance was detected in both univariable analyses $(-22(-26 ;-19) \vee-10(-15 ;-5)$ cells $/ \mathrm{mm}^{3} /$ year, interaction $\mathrm{p}<0.001$ ) and after both adjustments (Figure 2 ).

There was no evidence to suggest that CD4 cell decline differed according to the detection of PI resistance including minor PI mutations (Error! Reference source not found. 2). However, there was some weak evidence suggesting that CD4 cell decline was less steep during episodes where major PI mutations were detected, both in univariable (difference $=6(-1-12)$ cells $\left./ \mathrm{mm}^{3} / \mathrm{year}, \mathrm{p}=0.07\right)$ and 
multivariable models (difference $=7(1-14)$ cells $/ \mathrm{mm}^{3} /$ year, $p=0.02$ in model 1 , difference $=6(-0 ; 12)$ cells $/ \mathrm{mm}^{3} /$ year, $\mathrm{p}=0.05$ in model 2 ).

\section{The effect of specific DRM on CD4 count changes}

The difference in CD4 cell decline between episodes with and without a given RT mutation after adjustment for pre-specified confounders is shown in Figure 3. After correcting for multiple testing, the strongest association was found for M184V, with episodes where M184V was detected experiencing somewhat less steep CD4 cell declines (difference= 17 (11-23) cells/mm3/year, $\mathrm{q}<0.001$ ). These findings did not change markedly after additional adjustment for VL (difference $=16$ (10-21) cells/mm3/year, q<0.001, Supplementary Materials, S5). There was also reasonable evidence that the $\mathrm{T} 215 \mathrm{Y}$ and $\mathrm{D} 67 \mathrm{~N}$ mutations were associated with less steep CD4 cell declines both before (difference=11 (5-17) and $11(5-17)$ respectively, both $p=0.012)$ and after (difference=10 (416) and 12 (6-17), q=0.019 and 0.003 respectively) adjustment for VL. One NNRTI mutation, V179D, was associated with steeper CD4 declines, although the confidence intervals surrounding this finding were very wide (difference $=-44(-69 ;-19)$ cells $/ \mathrm{mm} 3 /$ year, $\mathrm{q}=0.012$, Figure 3 ). There was also some very weak evidence $(q=0.05)$ suggesting that those with the $L 74 V$ mutation experienced somewhat steeper CD4 declines, but the evidence supporting this finding disappeared upon adjustment for VL (Supplementary Materials, S5).

Of the PI mutations studied, 2 remained associated with a less steep CD4 decline after correction for multiple testing: V82A and I54V (Figure 4). The strongest association was found for the V82A mutation, where CD4 decline was estimated to be 18 (10-25) cells/mm3/year less steep during episodes where the mutation was detected compared to episodes without the mutation $(q<0.001)$. CD4 decline was also 13 (5-20) cells/mm3/year less steep during episodes where $154 \mathrm{~V}$ was detected $(q=0.015)$. Again, further adjustment for VL did not change these conclusions or shift the point estimates notably (Supplementary Materials, S5). 


\section{Clusters of mutations}

190

The PCA analysis identified five RT and two PR clusters. The process by which they were selected is described in more detail in the Supplementary Materials (S1). Briefly, the first three RT clusters corresponded roughly to the TAM mutations (RT codons $210,41,215,219,67,70$ plus 44, and 118), the $151 \mathrm{M}$ complex (RT codons 151, 116, 77and 75 and weakly, 65 and 62 ) and the TAM-2 pathway (RT codons 70, 219 and weakly, 67). The fourth RT cluster consisted primarily of substitutions at positions 74,221 and 181 and the fifth cluster of substitutions at positions 101,103 and 225 . Of the two PR clusters, the first consisted primarily of major PI mutations (codons $46,48,54,58,82,84$ and 90 ) with some minor PI mutations (codons $10,24,33,53,64,71$ and 73 ) which represented broad PI resistance. The second PI cluster consisted of 4 minor PI mutations (codons 20, 36, 69 and 89 ) which are common in non-B subtypes, with some weak contributions from substitutions in position 16 and 15.

\section{The effect of mutation clusters on CD4 count changes}

The effect of the mutation clusters on CD4 count changes are shown in Error! Reference source not found.. Individuals with scores in the highest quartile of the 3rd RT cluster (weakly corresponding to TAM-2 mutations) experienced reduced CD4 cell declines (difference $=9$ (2-16) cells $/ \mathrm{mm}^{3} /$ year, $\mathrm{p}=0.007)$, as did individuals with scores in the highest quartile of the $1^{\text {st }} \mathrm{PI}$ cluster, which represented broad PI resistance (difference $=14$ (7-20) cells $/ \mathrm{mm}^{3} /$ year, $\mathrm{p}<0.001$ ). Individuals with scores in the highest quartile of the $5^{\text {th }}$ RT cluster, which included K103N, experienced steeper CD4 cell declines (difference=-9 $(-16 ;-3)$ cells $/ \mathrm{mm}^{3} /$ year, $\left.\mathrm{p}=0.007\right)$ compared to individuals with lower scores on this component. There was also some very weak evidence that scores in the highest quartile of the $1^{\text {st }} \mathrm{RT}$ cluster, which included the TAM-1 mutations, were associated with somewhat reduced CD4 cell declines $(p=0.03)$. 


\section{Discussion}

216 Although the aim of HIV treatment should always be to suppress the VL to undetectable levels, there

217 is a minority of patients with extensive resistance or without access to any other drug options for whom this is not possible. Composing a salvage regimen for these individuals presents a particular challenge, and it has long been debated whether including drugs that preserve resistance patterns associated with lower viral fitness may provide some clinical benefit $[4,6,30]$. In this analysis, we found that among individuals maintained on a failing treatment regimen, CD4 counts declined less rapidly during episodes in which resistance was detected compared to episodes where resistance was not detected. DRM detection can serve as a proxy for ongoing ART pressure and at least partial antiviral activity, whereas the absence of DRM can indicate high levels of non-adherence. VF episodes with no detected DRM had much higher baseline VL levels in addition to more rapid CD4 cell decline, which supports the hypothesis that those individuals without DRM may not be taking their ART. When restricting the analyses to episodes with any detected resistance, CD4 counts decreased less steeply during episodes of virological failure where any NRTI resistance, the RT mutations M184V, D67N and T215Y and either of the PR mutations V82A and I54V were detected. CD4 counts declined more steeply during episodes with detected NNRTI resistance, as well as the RT mutations V179D and, marginally, L74V.

It has been suggested that differences in CD4 cell decline according to the presence of certain DRM could reflect differences in the replicative capacity of the virus [31]. The M184V mutation in the reverse transcriptase has been frequently shown to adversely impact replicative capacity [32-35], and the T215Y mutation has been associated with an impaired RC $[35,36]$. In contrast, D67N has been found to have relatively high $\mathrm{RC}[31,36]$. The V82A mutation has been reported to negatively impact the functionality of the viral protease which could lead to a lower RC [37]. However, adjustment for VL, which is associated with the replicative capacity of a virus [14], did not affect the 
conclusions or the size of the CD4 cell decline markedly in this analysis. Although this could indicate that the effects described here are not being mediated through viral load changes, a model adjusting for a mediating variable often fails to accurately estimate the total direct effect of the exposure on the outcome [38-40]. It is possible that adjusting for time-updated VL has not removed the effect that might be mediated through this variable, and this limitation should be born in mind when interpreting the results.

The results from studying clusters of mutations indicated that episodes where the mutation pattern broadly aligned with TAM-2 mutations or the broad PI resistance described by the $1^{\text {st }} \mathrm{PI}$ cluster had less steep CD4 cell declines. Although the TAM-2 mutations D67N, K70R and K219Q have relatively low fitness costs when present alone [31], the presence of K70R has been shown to result in reduction in fitness when it emerges in viruses carrying both D67N and K219Q in the absence of drug pressure [41]. Such a combined effect may also explain why the detection of each of these 3 mutations individually resulted in less steep CD4 cell declines, although only significantly so for the D67N mutation after correcting for multiple testing. The impact of PI resistance on replicative capacity is complex to predict as these mutations may be accompanied by compensatory PI mutations that can restore viral fitness [31]. However, it is possible that the first PI cluster could capture the effect of a number of major PI mutations which, when present in combination, could be associated with favourable CD4 cell changes. We also found weak evidence that the fourth RT cluster was associated with steeper CD4 cell declines. The mutations that formed part of this cluster include K103N and L100I, both of which have been shown to have a very small fitness cost [31].

Previous studies of the effect of drug resistance on CD4 cell changes have found conflicting results. A previous randomised trial, COLATE, which evaluated continuation versus discontinuation of lamivudine in individuals with VF as compared to other CART in order to study the effect of preserving the $\mathrm{M} 184 \mathrm{~V} / \mathrm{I}$ mutation, failed to demonstrate a virological or immunological benefit of 
continuing lamivudine [30]. The authors suggested that this could be due to the high GSS of the switch regimens, which could be masking any potential benefits. An earlier pilot trial (E-184V) reported a benefit of lamivudine monotherapy compared to treatment interruption in treatment experienced patients on HAART requesting a treatment interruption, and suggested that these benefits are in part due to the preservation of M184V [6]. Recently presented results from the MOBIDIP/ ANRS 12286 trial have also shown a marked clinical benefit of using lamivudine in combination with a boosted PI compared to the use of a boosted PI alone as a simplification strategy for patients with a suppressed VL on second line therapy [42]. The findings from observational studies have also been conflicting. In broad agreement with the findings presented here, a recent analysis by Hoffman et al of a large cohort of people living with HIV from South Africa found that CD4 counts declined less rapidly during episodes of persistent viraemia where M184V was detected, although not significantly so $(p=0.1)$ [43]. A study by Antinori et al in 2001 found that both M184V and V82A were associated with immunological recovery despite ongoing viral replication in univariable analyses, but not after adjustments for confounders including VL [20]. This is similar to results reported by Gianotti et al, who found no associations between individual mutations in the pol gene region and the odds of discordant immuno-virological responses when studying 825 individuals from the large ARCA database in Italy [19].

There are several important limitations of our findings. Firstly, we did not have a validated measure of adherence available, which unfortunately meant that we were not able to adjust for poor adherence. Adherence has the potential to be an important confounder in this analysis, as it is likely to influence both the presence of resistance and CD4 cell decline [44]. Although sensitivity analyses adherent individuals. Secondly, many different drug combinations were used to treat individuals in this study, and it was not possible to study the effect of individual regimens. Although adjustment 
for current VL should remove the effect of residual antiviral activity exerted through the VL, we cannot rule out that some of the different regimens used exerted some residual antiviral activity that could explain some of the findings. Although we conducted sensitive analyses where we adjusted for the class of drug received which showed consistent results (results available within the supplementary materials, S4), we cannot rule out that adjustment for the specific regimen received would produce different results due to the presence of residual confounding in our study. Future studies conducted in other settings may have greater homogeneity in the types of regimens used, and in these situations it would be of great interest to repeat the analyses stratified by the type of regimen received. In addition, we cannot rule out that the results would differ in settings where other subtypes predominate. Repeating the analyses within cohorts with a higher prevalence of nonB subtypes would be of great interest. Finally, despite using combined data from two large cohorts, the relatively low prevalence of individual mutations means that some of our estimates, particularly for rare mutations, suffer from low precision.

Bearing these limitations in mind, our findings provide some support for the hypothesis that CD4 cell decline may be less marked when individuals experiencing VF are maintained on regimens that preserve specific mutations that have previously been associated with reduced viral fitness. This could have implications for individuals in RLS who have failed all existing available lines, and suggests that in these instances the inclusion of lamivudine/emtricitabine and a boosted PI may be the best option in terms of composing a salvage regimen. However, the likely effect of the presence of a particular mutation on annual CD4 cell decline is likely to be relatively small and complex to predict due to the presence of epistatic interactions and potentially confounding effects which are hard to measure, such as residual drug action and adherence. Further research is needed to evaluate the clinical benefits of specific treatment strategies that aim to preserve particular resistance patterns among individuals for whom a potent ART regimen cannot be prescribed, as well as to elucidate the mechanisms through which any beneficial effect is mediated. Until then, efforts should focus on 
ensuring that those who experience VF have access to individualised care and a broad range of

319 antiretroviral drugs, and, where possible, novel ART drugs through compassionate early access

320 programs to allow the construction of fully suppressive regimens. 
321 


\section{References}

1. Imaz A, Falcó V, Ribera E. Antiretroviral salvage therapy for multiclass drug-resistant HIV-1infected patients: from clinical trials to daily clinical practice. AIDS Rev. 2011; 13(3):180-193.

2. WHO | Consolidated guidelines on the use of antiretroviral drugs for treating and preventing HIV infection [Internet]. WHO. [cited 2014 Jul 10]. Available from:

http://www.who.int/hiv/pub/guidelines/arv2013/en/

3. Phillips AN, Cohen C, Lundgren JD. Issues in the design of trials comparing management strategies for heavily pretreated patients. Curr Opin HIV AIDS. 2006; 1(6):476-481.

4. Deeks SG. Durable HIV treatment benefit despite low-level viremia: reassessing definitions of success or failure. JAMA. 2001; 286(2):224-226.

5. Ledergerber B. Predictors of trend in CD4-positive T-cell count and mortality among HIV-1infected individuals with virological failure to all three antiretroviral-drug classes. The Lancet. 2004; 364(9428):51-62.

6. Castagna A, Danise A, Menzo S, et al. Lamivudine monotherapy in HIV-1-infected patients harbouring a lamivudine-resistant virus: a randomized pilot study (E-184V study). AIDS Lond Engl. 2006; 20(6):795-803.

7. Campbell TB, Shulman NS, Johnson SC, et al. Antiviral activity of lamivudine in salvage therapy for multidrug-resistant HIV-1 infection. Clin Infect Dis Off Publ Infect Dis Soc Am. 2005; 41(2):236-242.

8. Deeks SG, Hoh R, Grant RM, et al. CD4+ T cell kinetics and activation in human immunodeficiency virus-infected patients who remain viremic despite long-term treatment with protease inhibitor-based therapy. J Infect Dis. 2002; 185(3):315-323.

9. Sloand EM, Kumar PN, Kim S, Chaudhuri A, Weichold FF, Young NS. Human immunodeficiency virus type 1 protease inhibitor modulates activation of peripheral blood CD4(+) T cells and decreases their susceptibility to apoptosis in vitro and in vivo. Blood. 1999; 94(3):1021-1027.

10. Stoddart CA, Liegler TJ, Mammano F, et al. Impaired replication of protease inhibitor-resistant HIV-1 in human thymus. Nat Med. 2001; 7(6):712-718.

11. Tang MW, Shafer RW. HIV-1 antiretroviral resistance: scientific principles and clinical applications. Drugs. 2012; 72(9):e1-25.

12. Cozzi-Lepri A, Paredes R, Phillips A, et al. The rate of accumulation of nonnucleoside reverse transcriptase inhibitor (NNRTI) resistance in patients kept on a virologically failing regimen containing an NNRTI*. HIV Med. 2012; 13(1):62-72.

13. Dionisio $D$, Vivarelli $A$, Zazzi $M$, et al. Long-term CD4+ T-cell response in HIV-1-infected patients continuing combination antiretroviral therapy despite high-level viremia and drug resistance. Eur J Clin Microbiol Infect Dis Off Publ Eur Soc Clin Microbiol. 2003; 22(1). 
14. Barbour JD, Wrin T, Grant RM, et al. Evolution of phenotypic drug susceptibility and viral replication capacity during long-term virologic failure of protease inhibitor therapy in human immunodeficiency virus-infected adults. J Virol. 2002; 76(21):11104-11112.

15. Vaidya NK, Rong L, Marconi VC, Kuritzkes DR, Deeks SG, Perelson AS. Treatment-mediated alterations in HIV fitness preserve CD4+ T cell counts but have minimal effects on viral load. PLoS Comput Biol. 2010; 6(11).

16. Renaud M, Katlama C, Mallet A, et al. Determinants of paradoxical CD4 cell reconstitution after protease inhibitor-containing antiretroviral regimen. AIDS Lond Engl. 1999; 13(6):669-676.

17. Rodes B, Garcia F, Gutierrez C, et al. Impact of drug resistance genotypes on CD4+ counts and plasma viremia in heavily antiretroviral-experienced HIV-infected patients. J Med Virol. 2005; 77(1):23-28.

18. Mendoza C de, Martin-Carbonero L, Gallego O, Corral A, Gonzalez-Lahoz J, Soriano V. Relationship between drug resistance mutations, plasma viremia, and CD4+ T-cell counts in patients with chronic HIV infection. J Med Virol. 2005; 76(1).

19. Gianotti N, Galli L, Zazzi M, et al. No pol mutation is associated independently with the lack of immune recovery in patients infected with HIV and failing antiretroviral therapy. J Med Virol. 2011; 83(3):391-398.

20. Antinori A, Liuzzi G, Cingolani A, et al. Drug-resistant mutants of HIV-1 in patients exhibiting increasing CD4 cell count despite virological failure of highly active antiretroviral therapy. AIDS Lond Engl. 2001; 15(17):2325-2327.

21. CHIP.DK > Ongoing Studies > EuroSIDA > About [Internet]. [cited 2015 Nov 16]. Available from: http://www.chip.dk/Ongoing-Studies/EuroSIDA/About

22. UKCHIC - Home [Internet]. [cited 2015 Nov 16]. Available from: http://128.40.115.16/UKCHIC/indexUKCHIC.asp

23. www.hivrdb.org.uk [Internet]. [cited 2015 Nov 16]. Available from: http://www.hivrdb.org.uk/

24. Wittkop L, Commenges D, Pellegrin I, et al. Alternative methods to analyse the impact of HIV mutations on virological response to antiviral therapy. BMC Med Res Methodol. 2008; 8:68.

25. Thiébaut $R$, Walker $S$. When it is better to estimate a slope with only one point. QJM. 2008; 101(10):821-824.

26. Singer JD. Using SAS PROC MIXED to Fit Multilevel Models, Hierarchical Models, and Individual Growth Models. J Educ Behav Stat. 1998; 23(4):323-355.

27. Glymour MM, Weuve J, Berkman LF, Kawachi I, Robins JM. When Is Baseline Adjustment Useful in Analyses of Change? An Example with Education and Cognitive Change. Am J Epidemiol.

2005; 162(3):267-278.

28. Calmy A, Balestre E, Bonnet F, et al. Mean CD4 cell count changes in patients failing a first-line antiretroviral therapy in resource-limited settings. BMC Infect Dis. 2012; 12:147.

29. Storey JD, Tibshirani R. Statistical significance for genomewide studies. Proc Natl Acad Sci. 2003; 100(16):9440-9445. 
30. Fox Z, Dragsted UB, Gerstoft J, et al. A randomized trial to evaluate continuation versus discontinuation of lamivudine in individuals failing a lamivudine-containing regimen: the COLATE trial. Antivir Ther. 2006; 11(6):761-770.

31. De Luca A. The impact of resistance on viral fitness and its clinical implications. In: Geretti AM, editor. Antiretrovir Resist Clin Pract [Internet]. London: Mediscript; 2006 [cited 2013 Aug 13]. Available from: http://www.ncbi.nlm.nih.gov/books/NBK2244/

32. Miller MD, White K, Petropoulos C, Parkin NT. Decreased Replication Capacity of HIV-1 Clinical Isolates Containing K65R or M184V RT Mutations (Poster 616). 10th Conf Retroviruses Opportunistic Infect. 2003; .

33. Wainberg MA. The impact of the M184V substitution on drug resistance and viral fitness. Expert Rev Anti Infect Ther. 2004; 2(1):147-151.

34. Back NK, Nijhuis M, Keulen W, et al. Reduced replication of 3TC-resistant HIV-1 variants in primary cells due to a processivity defect of the reverse transcriptase enzyme. EMBO J. 1996; 15(15):4040-4049.

35. Devereux HL, Emery VC, Johnson MA, Loveday C. Replicative fitness in vivo of HIV-1 variants with multiple drug resistance-associated mutations. J Med Virol. 2001; 65(2):218-224.

36. Prado JG, Franco S, Matamoros T, et al. Relative replication fitness of multi-nucleoside analogue-resistant HIV-1 strains bearing a dipeptide insertion in the fingers subdomain of the reverse transcriptase and mutations at codons 67 and 215. Virology. 2004; 326(1):103-112.

37. Menzo S, Monachetti A, Balotta C, et al. Processivity and drug-dependence of HIV-1 protease: determinants of viral fitness in variants resistant to protease inhibitors. AIDS Lond Engl. 2003; 17(5):663-671.

38. Robins JM, Greenland S. Identifiability and exchangeability for direct and indirect effects. Epidemiol Camb Mass. 1992; 3(2):143-155.

39. Kaufman JS, MacLehose RF, Kaufman S. A further critique of the analytic strategy of adjusting for covariates to identify biologic mediation. Epidemiol Perspect Innov. 2004; 1:4.

40. Cole SR, Hernán MA. Fallibility in estimating direct effects. Int J Epidemiol. 2002; 31(1):163165.

41. Cong M, Heneine W, García-Lerma JG. The Fitness Cost of Mutations Associated with Human Immunodeficiency Virus Type 1 Drug Resistance Is Modulated by Mutational Interactions. J Virol. 2007; 81(6):3037-3041.

42. Ciaffi L, Koulla-Shiro S, Sawadogo A, et al. Dual therapy with a boosted protease inhibitor plus lamivudine is an effective maintenance strategy in patients on second-line antiretroviral therapy in Africa: the ANRS 12286/MOBIDIP tria. Abstr HIV Glasg Suppl J Int AIDS Soc. 2016; 19(Suppl 7).

43. Hoffmann CJ, Maritz J, Zyl GU van. CD4 count-based failure criteria combined with viral load monitoring may trigger worse switch decisions than viral load monitoring alone. Trop Med Int Health TM IH. 2016; 21(2):219-223. 
44. Lucas GM. Antiretroviral adherence, drug resistance, viral fitness and HIV disease progression: a tangled web is woven. J Antimicrob Chemother. 2005; 55(4):413-416. 


\section{Transparency}

This work was presented in part at the HIV Glasgow Drug Therapy conference 2014, abstract P205.

Dr. Sabin reports grants from MRC during the conduct of the study; personal fees from Gilead Sciences, personal fees from ViiV Healthcare and personal fees from Janssen-Cilag outside the submitted work. Dr. Post reports grants and personal fees from ViiV Healthcare, grants and personal fees from Gilead Sciences, personal fees from Janssen, personal fees from MSD and personal fees from Abbvie outside the submitted work. No other author has reported any additional conflicts of interest.

\section{Funding and Acknowledgements}

\section{EuroSIDA}

EuroSIDA was supported by the European Union's Seventh Framework Programme for research, technological development and demonstration under EuroCoord grant agreement $n^{\circ}$ 260694. Current support includes unrestricted grants by Bristol-Myers Squibb, Gilead, GlaxoSmithKline LLC, Janssen R\&D, Merck and Co. Inc., Pfizer Inc. The participation of centres from Switzerland was supported by The Swiss National Science Foundation (Grant 108787). The study is also supported by a grant [grant number DNRF126] from the Danish National Research Foundation.

The multi-centre study group, EuroSIDA (national coordinators in parenthesis).Argentina: (M Losso), M Kundro, Hospital JM Ramos Mejia, Buenos Aires. Austria: (B Schmied), Pulmologisches Zentrum der Stadt Wien, Vienna; R Zangerle, Medical University Innsbruck, Innsbruck. Belarus: (I Karpov), A Vassilenko, Belarus State Medical University, Minsk, VM Mitsura, Gomel State Medical University, Gomel; D Paduto, Regional AIDS Centre, Svetlogorsk. Belgium: (N Clumeck), S De Wit, M Delforge, Saint-Pierre Hospital, Brussels; E Florence, Institute of Tropical Medicine, Antwerp; L Vandekerckhove, University Ziekenhuis Gent, Gent. Bosnia-Herzegovina: (V Hadziosmanovic), Klinicki Centar Univerziteta Sarajevo, Sarajevo. Croatia: (J Begovac), University Hospital of Infectious Diseases, Zagreb. Czech Republic: (L Machala), D Jilich, Faculty Hospital Bulovka, Prague; D Sedlacek, Charles University Hospital, Plzen. Denmark: G Kronborg,T Benfield, Hvidovre Hospital, Copenhagen; J Gerstoft, T Katzenstein, Rigshospitalet, Copenhagen; NF Møller, C Pedersen, Odense University Hospital, Odense; L Ostergaard, Skejby Hospital, Aarhus, L Wiese, Roskilde Hospital, Roskilde; L N Nielsen, Hillerod 
Hospital, Hillerod. Estonia: (K Zilmer), West-Tallinn Central Hospital, Tallinn; Jelena Smidt, Nakkusosakond Siseklinik, Kohtla-Järve. Finland: (M Ristola), I Aho, Helsinki University Central Hospital, Helsinki. France: (J-P Viard), Hôtel-Dieu, Paris; P-M Girard, Hospital Saint-Antoine, Paris; C Pradier, E Fontas, Hôpital de l'Archet, Nice; C Duvivier, Hôpital Necker-Enfants Malades, Paris.Germany: (J Rockstroh), Universitäts Klinik Bonn; R Schmidt, Medizinische Hochschule Hannover; O Degen, University Medical Center Hamburg-Eppendorf, Infectious Diseases Unit, Hamburg; HJ Stellbrink, IPM Study Center, Hamburg; C Stefan, JW Goethe University Hospital, Frankfurt; J Bogner, Medizinische Poliklinik, Munich; G. Fätkenheuer, Universität Köln, Cologne. Georgia: (N Chkhartishvili) Infectious Diseases, AIDS \& Clinical Immunology Research Center, TbilisiGreece: P Gargalianos, G Xylomenos, P Lourida, Athens General Hospital; H Sambatakou, Ippokration General Hospital, Athens. Hungary: (J Szlávik), Szent Lásló Hospital, Budapest. Iceland: (M Gottfredsson), Landspitali University Hospital, Reykjavik. Ireland: (F Mulcahy), St. James's Hospital, Dublin.Israel: (I Yust), D Turner, M Burke, Ichilov Hospital, Tel Aviv; E Shahar, G Hassoun, Rambam Medical Center, Haifa; H Elinav, M Haouzi, Hadassah University Hospital, Jerusalem; D Elbirt, ZM Sthoeger, AIDS Center (Neve Or), Jerusalem. Italy: (A D’Arminio Monforte), Istituto Di Clinica Malattie Infettive e Tropicale, Milan; R Esposito, I Mazeu, C Mussini, Università Modena, Modena; F Mazzotta, A Gabbuti, Ospedale S Maria Annunziata, Firenze; V Vullo, M Lichtner, University di Roma la Sapienza, Rome; M Zaccarelli, A Antinori, R Acinapura, M Plazzi, Istituto Nazionale Malattie Infettive Lazzaro Spallanzani, Rome; A Lazzarin, A Castagna, N Gianotti, Ospedale San Raffaele, Milan; M Galli, A Ridolfo, Osp. L. Sacco, Milan.Latvia: (B Rozentale), Infectology Centre of Latvia, Riga. Lithuania: (V Uzdaviniene) Vilnius University Hospital Santariskiu Klinikos, Vilnius; R Matulionyte, Center of Infectious Diseases, Vilnius University Hospital Santariskiu Klinikos, Vilnius. Luxembourg: (T Staub), R Hemmer, Centre Hospitalier, Luxembourg. Netherlands: (P Reiss), Academisch Medisch Centrum bij de Universiteit van Amsterdam, Amsterdam.Norway: (V Ormaasen), A Maeland, J Bruun, Ullevål Hospital, Oslo. Poland: (B Knysz), J Gasiorowski, M Inglot, Medical University, Wroclaw; A Horban, E Bakowska, Centrum Diagnostyki i Terapii AIDS, Warsaw; R Flisiak, A Grzeszczuk, Medical University, Bialystok; M Parczewski, M Pynka, K Maciejewska, Medical Univesity, Szczecin; M Beniowski, E Mularska, Osrodek Diagnostyki i Terapii AIDS, Chorzow; T Smiatacz, M Gensing, Medical University, Gdansk; E Jablonowska, E Malolepsza, K Wojcik, Wojewodzki Szpital Specjalistyczny, Lodz; I Mozer-Lisewska, Poznan University of Medical Sciences, Poznan.Portugal: (L Caldeira), Hospital Santa Maria, Lisbon; K Mansinho, Hospital de Egas Moniz, Lisbon; F Maltez, Hospital Curry Cabral, Lisbon. Romania: (R Radoi), C Oprea, Spitalul de Boli Infectioase si Tropicale: Dr. 
Victor Babes, Bucarest. Russia: (A Panteleev), O Panteleev, St Petersburg AIDS Centre, St Peterburg; A Yakovlev, Medical Academy Botkin Hospital, St Petersburg; T Trofimora, Novgorod Centre for AIDS, Novgorod, I Khromova, Centre for HIV/AIDS \& and Infectious Diseases, Kaliningrad; E Kuzovatova, Nizhny Novgorod Scientific and Research Institute of Epidemiology and Microbiology named after Academician I.N. Blokhina, Nizhny Novogrod; E Borodulina, E Vdoushkina, Samara State Medical University, Samara.Serbia: (D Jevtovic), The Institute for Infectious and Tropical Diseases, Belgrade. Slovenia: (J Tomazic), University Clinical Centre Ljubljana, Ljubljana. Spain: (JM Gatell), JM Miró, Hospital Clinic Universitari de Barcelona, Barcelona; S Moreno, J. M. Rodriguez, Hospital Ramon y Cajal, Madrid; B Clotet, A Jou, R Paredes, C Tural, J Puig, I Bravo, Hospital Germans Trias i Pujol, Badalona; P Domingo, M Gutierrez, G Mateo, MA Sambeat, Hospital Sant Pau, Barcelona; JM Laporte, Hospital Universitario de Alava, Vitoria-Gasteiz. Sweden: (K Falconer), A Thalme, A Sonnerborg, Karolinska University Hospital, Stockholm; A Blaxhult, Venhälsan-Sodersjukhuset, Stockholm; L Flamholc, Malmö University Hospital, Malmö. Switzerland: (A Scherrer), R Weber, University Hospital Zurich; M Cavassini, University Hospital Lausanne; A Calmy, University Hospital Geneva; H Furrer, University Hospital Bern; M Battegay, University Hospital Basel; P Schmid, Cantonal Hospital St. Gallen.Ukraine: A Kuznetsova, Kharkov State Medical University, Kharkov; G Kyselyova, Crimean Republican AIDS centre, Simferopol; M Sluzhynska, Lviv Regional HIV/AIDS Prevention and Control CTR, Lviv.United Kingdom: (B Gazzard), St. Stephen's Clinic, Chelsea and Westminster Hospital, London; AM Johnson, E Simons, S Edwards, Mortimer Market Centre, London; A Phillips, MA Johnson, A Mocroft, Royal Free and University College Medical School, London (Royal Free Campus); C Orkin, Royal London Hospital, London; J Weber, G Scullard, Imperial College School of Medicine at St. Mary's, London; A Clarke, Royal Sussex County Hospital, Brighton; C Leen, Western General Hospital, Edinburgh.The following centers have previously contributed data to EuroSIDA:Infectious Diseases Hospital, Sofia, Bulgaria Hôpital de la Croix Rousse, Lyon, France Hôpital de la Pitié-Salpétière, Paris, France Unité INSERM, Bordeaux, France Bernhard Nocht Institut für Tropenmedizin, Hamburg, Germany 1st I.K.A Hospital of Athens, Athens, Greece Ospedale Riuniti, Divisione Malattie Infettive, Bergamo, Italy Ospedale di Bolzano, Divisione Malattie Infettive, Bolzano, Italy Ospedale Cotugno, III Divisione Malattie Infettive, Napoli, Italy Dérer Hospital, Bratislava, Slovakia Hospital Carlos III, Departamento de Enfermedades Infecciosas, Madrid, Spain Kiev Centre for AIDS, Kiev, Ukraine Luhansk State Medical University, Luhansk, Ukraine Odessa Region AIDS Center, Odessa, Ukraine 
EuroSIDA Steering Committee: J Gatell, B Gazzard, A Horban, I Karpov, B Ledergerber, M Losso, A d'Arminio Monforte, C Pedersen, M Ristola, A Phillips, P Reiss, J Lundgren, J Rockstroh Chair: J Rockstroh Study Coleads: A Mocroft, O Kirk EuroSIDA Coordinating Centre Staff: O Kirk, L Peters, C Matthews, AH Fischer, A Bojesen, D Raben, D Kristensen, K Grønborg Laut, JF Larsen, D Podlekareva Statistical Staff: A Mocroft, A Phillips, A Cozzi-Lepri, L Shepherd, A Schultze

\section{UK CHIC}

Steering Committee: Jonathan Ainsworth, Sris Allan, Jane Anderson, Abdel Babiker, David Chadwick, Valerie Delpech, David Dunn, Martin Fisher, Brian Gazzard, Richard Gilson, Mark Gompels, Phillip Hay, Teresa Hill, Margaret Johnson, Sophie Jose, Stephen Kegg, Clifford Leen, Fabiola Martin, Mark Nelson, Chloe Orkin, Adrian Palfreeman, Andrew Phillips, Deenan Pillay, Frank Post, Jillian Pritchard, Caroline Sabin, Memory Sachikonye, Achim Schwenk, Anjum Tariq, John Walsh.

Central Co-ordination: University College London (Teresa Hill, Sophie Jose, Andrew Phillips, Caroline Sabin, Alicia Thornton); Medical Research Council Clinical Trials Unit at UCL (MRC CTU at UCL), London (David Dunn, Adam Glabay).

Participating Centres: Barts Health NHS Trust, London (Chloe Orkin, Janet Lynch, James Hand, Carl de Souza); Brighton and Sussex University Hospitals NHS Trust (Martin Fisher, Nicky Perry, Stuart Tilbury, Elaney Youssef, Duncan Churchill); Chelsea and Westminster Hospital NHS Foundation Trust, London (Brian Gazzard, Mark Nelson, Rhiannon Everett, David Asboe, Sundhiya Mandalia); Public Health England, London (Valerie Delpech); Homerton University Hospital NHS Trust, London (Jane Anderson, Sajid Munshi); King's College Hospital NHS Foundation Trust, London (Frank Post, Ade Adefisan, Chris Taylor, Zachary Gleisner, Fowzia Ibrahim, Lucy Campbell); Medical Research Council Clinical Trials Unit (MRC CTU), London (Abdel Babiker, David Dunn, Adam Glabay); Middlesbrough, South Tees Hospitals NHS Foundation Trust, (David Chadwick, Kirsty Baillie); Mortimer Market Centre, University College London (Richard Gilson, Nataliya Brima, Ian Williams); North Middlesex University Hospital NHS Trust, London (Jonathan Ainsworth, Achim Schwenk, Sheila Miller, Chris Wood); Royal Free NHS Foundation Trust/University College London (Margaret Johnson, Mike Youle, Fiona Lampe, Colette Smith, Rob Tsintas, Clinton Chaloner, Samantha Hutchinson, Caroline Sabin, Andrew Phillips 
Teresa Hill, Sophie Jose, Alicia Thornton, Susie Huntington); Imperial College Healthcare NHS Trust, London (John Walsh, Nicky Mackie, Alan Winston, Jonathan Weber, Farhan Ramzan, Mark Carder); The Lothian University Hospitals NHS Trust, Edinburgh (Clifford Leen, Alan Wilson, Sheila Morris); North Bristol NHS Trust (Mark Gompels, Sue Allan); Leicester, University Hospitals of Leicester NHS Trust (Adrian Palfreeman, Khurram Memon, Adam Lewszuk); Woolwich, Lewisham and Greenwich NHS Trust (Stephen Kegg, Akin Faleye, Dr Mitchell, Dr Hunter), UK Community Advisory Board (Memory Sachikonye); St. George's Healthcare NHS Trust (Phillip Hay, Mandip Dhillon, Christian Kemble); York Teaching Hospital NHS Foundation Trust (Fabiola Martin, Sarah Russell-Sharpe, Janet Gravely); Coventry, University Hospitals Coventry and Warwickshire NHS Trust (Sris Allan, Andrew Harte, Stephen Clay); Wolverhampton, The Royal Wolverhampton Hospitals NHS Trust (Anjum Tariq, Hazel Spencer, Ron Jones); Chertsey, Ashford and St.Peter's Hospitals NHS Foundation Trust (Jillian Pritchard, Shirley Cumming, Claire Atkinson).

Funding: UK CHIC is funded by the UK Medical Research Council (Grant numbers G0000199, G0600337, G0900274 and M004236). The views expressed in this manuscript are those of the researchers and not necessarily those of the Medical Research Council.

\section{UK HIV Drug Resistance Database}

Steering Committee: Celia Aitken (Gartnavel General Hospital, Glasgow); David Asboe, Anton Pozniak (Chelsea \& Westminster Hospital, London); Patricia Cane (Public Health England, Porton Down); David Chadwick (South Tees Hospitals NHS Trust, Middlesbrough); Duncan Churchill (Brighton and Sussex University Hospitals NHS Trust); Duncan Clark (St Bartholomew's and The London NHS Trust); Simon Collins (HIV i-Base, London); Valerie Delpech (Centre for Infections, Public Health England); Samuel Douthwaite (Guy's and St. Thomas' NHS Foundation Trust, London); David Dunn, Esther Fearnhill, Kholoud Porter, Anna Tostevin, Ellen White, Oliver Stirrup (Research Department of Infection and Population Health, UCL); Christophe Fraser (Imperial College London); Anna Maria Geretti (Institute of Infection and Global Health, University of Liverpool); Antony Hale (Leeds Teaching Hospitals NHS Trust); Stéphane Hué (University College London); Steve Kaye (Imperial College, London); Paul Kellam (Wellcome Trust Sanger Institute \& University College London Medical School); Linda Lazarus (Expert Advisory Group on AIDS Secretariat, Public Health England); Andrew Leigh-Brown (University of Edinburgh); Tamyo Mbisa (Virus Reference Department, Public Health England); Nicola Mackie (Imperial NHS 
Trust, London); Samuel Moses (King's College Hospital, London); Chloe Orkin (St. Bartholomew's Hospital, London); Eleni Nastouli, Deenan Pillay, Andrew Phillips, Caroline Sabin (University College London, London); Erasmus Smit (Public Health England, Birmingham Heartlands Hospital); Kate Templeton (Royal Infirmary of Edinburgh); Peter Tilston (Manchester Royal Infirmary); lan Williams (Mortimer Market Centre, London); Hongyi Zhang (Addenbrooke's Hospital, Cambridge).

Coordinating Centre: Research Department of Infection and Population Health, UCL (David Dunn, Keith Fairbrother, Esther Fearnhill, Kholoud Porter, Anna Tostevin, Ellen White, Oliver Stirrup)

Centres contributing data: Clinical Microbiology and Public Health Laboratory, Addenbrooke's Hospital, Cambridge (Jane Greatorex); Guy's and St. Thomas' NHS Foundation Trust, London (Siobhan O’Shea, Jane Mullen); PHE - Public Health Laboratory, Birmingham Heartlands Hospital, Birmingham (Erasmus Smit); PHE Virus Reference Department, London (Tamyo Mbisa); Imperial College Health NHS Trust, London (Alison Cox); King's College Hospital, London (Richard Tandy); Medical Microbiology Laboratory, Leeds Teaching Hospitals NHS Trust (Tracy Fawcett); Specialist Virology Centre, Liverpool (Mark Hopkins, Lynne Ashton); Department of Clinical Virology, Manchester Royal Infirmary, Manchester (Peter Tilston); Department of Virology, Royal Free Hospital, London (Claire Booth, Ana Garcia-Diaz); Edinburgh Specialist Virology Centre, Royal Infirmary of Edinburgh (Lynne Renwick); Department of Infection \& Tropical Medicine, Royal Victoria Infirmary, Newcastle (Matthias L Schmid, Brendan Payne); South Tees Hospitals NHS Trust, Middlesbrough (David Chadwick); Department of Virology, St Bartholomew's and The London NHS Trust (Jonathan Hubb); Molecular Diagnostic Unit, Imperial College, London (Steve Kaye); University College London Hospitals (Stuart Kirk); West of Scotland Specialist Virology Laboratory, Gartnavel, Glasgow (Rory Gunson, Amanda Bradley-Stewart, Celia Aitken).

Funding: This work is currently supported by the UK Medical Research Council (Award Number 164587). 
